\section{RSP}

http://www.rsp.fsp.usp.br/
Revista de Saúde Pública

\title{
Eventos adversos de vacinas e as consequências da não vacinação: uma análise crítica
}

Luana Raposo de Melo Moraes Aps', Marco Aurélio Floriano Piantola', Sara Araujo Pereira', Julia Tavares de Castro"', Fernanda Ayane de Oliveira Santos'"', Luís Carlos de Souza Ferreira'

I Universidade de São Paulo. Instituto de Ciências Biomédicas. Departamento de Microbiologia. São Paulo, SP, Brasil

" Instituto Butantan. Centro de Biotecnologia. São Paulo, SP, Brasil

III Instituto Adolfo Lutz. Centro de Imunologia. São Paulo, SP, Brasil

\section{RESUMO}

OBJETIVO: Analisar os riscos relacionados às vacinas e os impactos da não vacinação para a população mundial.

MÉTODOS: Revisão narrativa que considerou informações contidas nas bases de dados bibliográficos NCBI-PubMed, Medline, Lilacs e Scientific Electronic Library Online (SciELO), no período compreendido entre novembro de 2015 e novembro de 2016. Para a análise de surtos ocasionados pela não vacinação foram considerados os trabalhos publicados entre 2010 e 2016.

RESULTADOS: Foram descritos os principais componentes das vacinas oferecidas pelo sistema público de saúde brasileiro e eventos adversos associados a esses elementos. Com exceção de reações inflamatórias locais e efeitos raros como exacerbação de doenças autoimunes e alergias, não foi demonstrada relação causal entre a administração de vacinas e autismo, mal de Alzheimer ou narcolepsia. Por outro lado, a falta de informações e a divulgação de informações não científicas têm contribuído para a reemergência de doenças infecciosas em diversos países no mundo e põe em risco planos globais para a erradicação de doenças infecciosas.

CONCLUSÕES: A população deve estar bem informada quanto aos benefícios da vacinação e os profissionais da saúde devem assumir o papel de divulgar informações verídicas e com respaldo científico sobre o tema, como compromisso ético e profissional junto à sociedade.

DESCRITORES: Vacinação, utilização. Vacinação, efeitos adversos. Vacinas. Imunização. Adjuvantes Imunológicos, contraindicações. Fatores de Risco. Controle de Doenças Transmissíveis.

Recebido: 29 nov 2016

Aprovado: 28 abr 2017

Como citar: Aps LRMM

Piantola MAF, Pereira SA, Castro

JT, Santos FAO, Ferreira LCS.

Eventos adversos de vacinas e as

consequências da não vacinação:

uma análise crítica. Rev Saude

Publica. 2018;52:40.

Copyright: Este é um artigo de acesso aberto distribuído sob os termos da Licença de Atribuição Creative Commons, que permite uso irrestrito, distribuição e reprodução em qualquer meio desde que $o$ autor e a fonte originais sejam creditados. 


\section{INTRODUÇÃO}

A primeira vacina foi descoberta por Edward Jenner em 1796, após 20 anos de estudos e experimentos com a varíola bovina, dando origem aos termos vaccine e vaccination (derivados do termo latino vacca). Na Inglaterra do século XVIII, a varíola era responsável por cerca de $10 \%$ dos óbitos totais e um terço das mortes era registrado entre as crianças. Classificada como uma das doenças mais devastadoras da história da humanidade, a varíola foi considerada erradicada pela Organização Mundial de Saúde (OMS) em 1980, após realização de um programa de vacinação em massa de ordem mundial ${ }^{1}$.

Apesar da notória relevância na erradicação ou controle de diversas doenças infectocontagiosas, as vacinas estão frequentemente relacionadas a questionamentos e críticas sobre efeitos adversos. Também já estiveram envolvidas em alguns eventos trágicos da indústria farmacêutica. O maior deles ocorreu em 1955, após falha no processo de fabricação da vacina contra a poliomielite baseada em vírus inativados ${ }^{2}$. Outros episódios foram registrados envolvendo componentes específicos das vacinas BCG (Bacillus Calmette-Guérin), tríplice viral (sarampo, caxumba e rubéola), rotavírus ${ }^{3}$, poliomielite oral ${ }^{4}$ e pertussis celular ${ }^{5}$. Em virtude de tais eventos, esforços foram investidos para garantir maior segurança na fabricação e uso de vacinas e solucionaram definitivamente problemas como aqueles mencionados acima. A formulação baseada em vírus inativado (conhecida por Salk ou VIP) é atualmente administrada em crianças de até quatro meses. Por conter vírus mortos, evitam os efeitos adversos graves observados com a formulação com vírus atenuado (VOP). Outro exemplo é a vacina contra a coqueluche (presente na vacina dTpa - difteria, tétano e pertussis acelular), que sofreu modificações para substituir a vacina pertussis celular, relacionada a eventos adversos graves na década de $1970^{6}$.

A criação do Programa Nacional de Imunizações (PNI), em 1973, por determinação do Ministério da Saúde representou um avanço de grande importância para a saúde pública no Brasil. Atualmente, 19 vacinas recomendadas pela OMS são oferecidas gratuitamente no Sistema Único de Saúde (SUS) e beneficiam todas as faixas etárias, seguindo um calendário nacional de vacinação ${ }^{7}$. Com o objetivo de coordenar as ações de imunização, o programa garantiu a continuidade de aplicação de doses (cumprimento do cronograma) e ampliou a área de cobertura vacinal no Brasil ${ }^{8}$, alcançando médias superiores a 95\% de cobertura vacinal para o calendário infantil ${ }^{9}$. Alguns resultados importantes são a eliminação da poliomielite e da transmissão sustentada do sarampo e da rubéola no país?.

No entanto, apesar do impacto na redução de casos e mortes pelas doenças imunopreveníveis, movimentos antivacinação são cada vez mais frequentes e persuasivos. Esses movimentos utilizam estratégias como distorção e divulgação de informações falsas que, alegando uma base científica, questionam a eficácia e segurança de diversas vacinas ${ }^{10}$. Em sua maioria, tais relatos relacionam vacinas, como a tríplice viral, adjuvantes e o conservante timerosal com a ocorrência de autismo em crianças. Busca-se uma associação temporal, principalmente pelo fato da doença ser diagnosticada no período posterior à aplicação da maioria das vacinas do calendário infantil, sem necessariamente haver uma relação causal. Outro exemplo de associação temporal sem base causal recentemente divulgada na mídia foi a ocorrência de casos de paralisia temporária após a imunização com a vacina contra o vírus papiloma humano (HPV).

As vacinas são rigorosamente testadas e monitoradas pelos seus fabricantes e pelos sistemas de saúde dos países onde são aplicadas. O licenciamento e a comercialização de vacinas ocorrem após aprovação de órgãos reguladores específicos e estudos clínicos cuidadosos, caros e demorados (ensaios de fase I, II, e III), com voluntários credenciados. A fase IV ocorre somente após a aprovação da comercialização do produto e tem como objetivo principal detectar eventos adversos não registrados nas fases anteriores, os chamados eventos adversos pós-vacinação (EAPV). A OMS recomendou a vigilância de EAPV a partir de 1991, e foi estruturado o Sistema Nacional de Vigilância dos Eventos Adversos Pós-Vacinação (VEAPV) no Brasil em 1992. Além disso, o Instituto Nacional de Controle de Qualidade em Saúde 
(INCQS), diretamente articulado com o Sistema Nacional de Vigilância Sanitária, garante a qualidade dos imunobiológicos distribuídos, cujas taxas de rejeição são inferiores a $1 \%^{4}$.

Uma rápida pesquisa com o termo "anti-vaccination" em uma das maiores redes sociais atualmente utilizadas apontou 20 páginas e 17 grupos relacionados a movimentos antivacinação, com quase 15 mil seguidores em um deles. O mesmo termo foi aplicado no maior site de buscas da web, que apresentou mais de seis milhões de resultados, dentre eles, diversos blogs e comunidades que apoiam a não vacinação. Isso mostra a necessidade de esclarecer a população sobre a importância das vacinas e o perigo representado pela não vacinação.

O presente estudo teve por objetivo avaliar os possíveis riscos associados a vacinas em uso no Brasil.

\section{MÉTODOS}

Estudo com base em buscas na literatura quanto à investigação de riscos associados à vacinação e a surtos desencadeados pela prática da não vacinação e assuntos relacionados. Trata-se de uma revisão narrativa, que considerou informações contidas nas seguintes bases de dados bibliográficos: PubMed, Medline, Lilacs e Scientific Electronic Library Online (SciELO). Sobre a investigação dos riscos associados às vacinas e seus componentes, a busca foi realizada entre novembro de 2015 e novembro de 2016 usando os descritores: EAPV, eventos adversos, vacina, vacinação e termos referentes a uma doença, vacina ou componente específico, como autismo, síndrome, vacina de HPV, vacina tríplice viral, timerosal. Os mesmos termos, na língua inglesa, também foram utilizados: "adverse", "events", "effects", "vaccine", "vaccination", "autism", "syndrome", "hpv vaccine", "MMR vaccine", "thimerosal". Para a análise de surtos ocasionados pela não vacinação foi realizada a busca na plataforma NCBI-PubMed com os termos que referenciavam as doenças com vacinação disponível, por exemplo, "measles", "mumps", adicionado aos termos "outbreak" e "unvaccinated". Os resultados foram selecionados após a leitura do resumo do artigo e foram considerados apenas os artigos que apresentavam relação clara entre eventos de não vacinação e surtos ou epidemias da doença, publicados entre 2010 e 2016. Foi feito um levantamento sobre o tema utilizando os termos "anti-vaccine movement", "vaccine hesitancy", "vaccine refusal" e "non-vaccination" adicionados ou não ao termo "outbreak". Artigos, cartas, resumos ou dissertações em outros idiomas, além do português, inglês ou espanhol foram excluídos.

\section{RESULTADOS}

\section{Riscos Associados à Vacinação}

A maioria das notícias vinculadas em mídias sociais informais e alguns trabalhos publicados apresentaram ou sugeriram o autismo ou TEA (Transtorno do Espectro Autista) como uma das principais doenças atribuídas à prática da vacinação, principalmente, à vacina tríplice viral (sarampo-caxumba-rubéola), também conhecida como vacina MMR (do termo em inglês "Measles, Mumps, and Rubella"). Porém, a Anvisa (Agência Nacional de Vigilância Sanitária), assim como o FDA (Food and Drug Administration), principal órgão regulador dos Estados Unidos, não comprovaram qualquer associação entre vacinas e o aumento dos casos de autismo na população $0^{2,11-13}$.

Os adjuvantes, frequentemente encontrados nas formulações vacinais, também podem estar associados ao aparecimento de reações e eventos adversos. Dentre os adjuvantes utilizados na produção das vacinas, destacam-se os sais minerais (sais de alumínio e sais de cálcio), os derivados microbianos, como o Monofosforil Lipídio A (MPL) e emulsões óleo em água empregando o esqualeno como composto principal (AS03 e MF59) ${ }^{14}$. A administração desses compostos pode provocar reações adversas ${ }^{8}$, como reações inflamatórias locais e, com frequência muito menor, efeitos sistêmicos, como a exacerbação de doenças autoimunes 
e alergias. Além dessas, outras doenças foram investigadas quanto à relação causal com os adjuvantes ${ }^{15}$. Destacaram-se as reações aos sais de alumínio, como alergias e ASIA (do termo em inglês "Autoimmune (inflammatory) Syndrome Induced by Adjuvants") ${ }^{15,16}$, miofascite macrofágica ${ }^{15,17}$ e doenças neurológicas, como por exemplo, o Alzheimer ${ }^{18} \mathrm{e}$ as síndromes incluídas no TEA ${ }^{18}$. No entanto, em nenhuma delas foi relatada uma correlação a partir de análises científicas. Além dos sais de alumínio, destacaram-se também as doenças relacionadas ao esqualeno, contido nas vacinas da influenza pandêmica e sazonal, como a Síndrome da Guerra do Golfo ${ }^{15,18}$ e Narcolepsia ${ }^{19,20}$ (Tabela 1), porém nenhuma relação de causalidade foi encontrada.

Além dos adjuvantes, outros componentes vacinais, como estabilizantes e conservantes, podem estar relacionados a diferentes eventos adversos (Tabela 1). Os principais exemplos são: a albumina e a gelatina (proteínas utilizadas como estabilizantes); antibióticos, comumente utilizados durante as primeiras etapas da preparação vacinal e frequentemente associados a reações alérgicas; e o formaldeído, que em forma líquida, é utilizado nas etapas iniciais de algumas vacinas como agente inativador de toxinas ou partículas virais. $\mathrm{O}$ formaldeído tem sido relacionado a alguns eventos adversos como eczema e até câncer. Entretanto, os estudos que avaliaram a associação de ocorrência de câncer com o uso de formaldeído comprovaram a associação após exposição a grandes quantidades ou pela exposição frequente, ou seja, em condições que não se aplicam a vacinas ${ }^{29,30}$. Proteínas de ovo também podem estar presentes em quantidades muito baixas em algumas vacinas que utilizam vírus cultivados em ovos embrionados, como a vacina contra a influenza. Essas proteínas podem desencadear uma resposta alérgica em pessoas intolerantes a esse componente ${ }^{31,32}$.

Utilizado como conservante em algumas vacinas licenciadas, o timerosal é um composto orgânico a base de mercúrio que também já esteve envolvido em questões polêmicas sobre a segurança das vacinas. A associação entre autismo, mercúrio e vacinas surgiu com a publicação de um trabalho em 1998, pelo médico inglês Andrew Wakefield, em uma das revistas mais importantes do ramo científico ${ }^{33}$. Nesse estudo, os autores apontaram sintomas, como desordens intestinais e atraso de desenvolvimento em 12 crianças avaliadas, e alterações de comportamento (incluindo autismo) em nove delas. Em 2010, após decisão judicial, o artigo foi inteiramente retratado após terem sido descobertas informações falsas contidas no estudo ${ }^{34} \mathrm{e}$ acordos de pagamentos envolvendo o pesquisador e advogados em processos por compensação de danos vacinais. Alguns trabalhos mostraram ainda que a dose de mercúrio ingerida normalmente por um indivíduo na alimentação é muito maior que a quantidade presente nas vacinas ${ }^{35-38}$. Até hoje, nenhum órgão regulamentador comprovou, de fato, a associação entre essas doenças e o conservante.

Tabela 1. Eventos adversos associados ao uso de adjuvantes vacinais.

\begin{tabular}{|c|c|c|c|}
\hline Adjuvante & Vacina & Eventos adversos estudados & Referências \\
\hline \multirow{2}{*}{ Sais de alumínio } & $\begin{array}{c}\text { Tríplice bacteriana (DTP) } \\
\text { Pentavalente } \\
\text { Hepatite A e B } \\
\text { Meningocóccica }\end{array}$ & $\begin{array}{c}\text { Miofascite macrofágica; } \\
\text { Alzheimer e TEA }\end{array}$ & 17,21 \\
\hline & HPV & $\begin{array}{c}\text { Síndrome Postural Ortostática } \\
\text { Taquicardizante (SPOT); } \\
\text { Síndrome de Guillain-Barré (SGB) }\end{array}$ & $22-25$ \\
\hline Esqualeno - MF59 & $\begin{array}{c}\text { HIV; Herpes zóster; } \\
\text { Citomegalovirus; Influenza }\end{array}$ & Síndrome da Guerra do Golfo & 8,26 \\
\hline Esqualeno - ASO3 & Influenza & $\begin{array}{l}\text { Narcolepsia } \\
\text { Parestesia }\end{array}$ & $8,27,28$ \\
\hline
\end{tabular}


De forma geral, a ocorrência de reações de hipersensibilidade depende de fatores de susceptibilidade, que torna o indivíduo predisposto à sua ocorrência. Desta maneira, a administração de certas vacinas é contraindicada em pacientes com história de reação anafilática ao leite, ovo ou qualquer outro componente que esteja presente em uma determinada formulação. Há também evidências de que alguns eventos adversos decorrem de fatores genéticos, como narcolepsia relacionada à vacina de influenza (tendo como adjuvante esqualeno e alfatocoferol). Outros eventos adversos são considerados como idiossincrásicos, ou seja, dependentes de fatores individuais ${ }^{8}$.

Recentemente, as vacinas profiláticas contra infecção por HPV ganharam espaço na mídia pelo possível envolvimento com a Síndrome de Guillain-Barré (SGB) e a Síndrome Postural Ortostática Taquicardizante (SPOT). A SGB é uma doença autoimune que causa danos no sistema nervoso e provoca sensação de formigamento, fraqueza muscular e até mesmo paralisia (Tabela 2). Em geral, manifesta-se após vacinação com formulações contendo vetores virais $^{4}$ ou, neste caso, VLP (do termo em inglês "Viral Like Particles") utilizado nas vacinas contra HPV atualmente comercializadas. A SPOT também é uma síndrome que acarreta em danos nervosos, porém provoca sintomas um pouco mais brandos como: palpitações, mal-estar e tonturas (Tabela 2). De fato, alguns trabalhos mostraram associação temporal entre vacinas e SGB/SPOT ${ }^{24,25}$. Entretanto, a OMS informa que nenhum evento adverso grave foi constatado, mesmo após sua aplicação em milhões de pessoas, e que a ocorrência de SGB em pessoas vacinadas possui frequência similar aos casos de doença com causa desconhecida ${ }^{41}$. A comercialização dessas vacinas continua liberada pela Anvisa e pelo FDA, e a constatação de eventuais efeitos adversos deve ser comunicada aos órgãos de saúde ou aos responsáveis pela distribuição e aplicação das vacinas.

Outra formulação vacinal que merece destaque em relação à possível ocorrência de efeitos adversos é a vacina contra a dengue, recentemente licenciada para uso no Brasil. Conhecida por "CYD-TDV" ou “Dengvaxia", a vacina é baseada em quatro linhagens de vírus vivos atenuados de composição quimérica, isto é, composta pelo vírus da febre amarela, expressando proteínas de envelope dos quatro sorotipos de DENV ${ }^{42}$. A formulação foi submetida, paralelamente, a dois estudos clínicos de fase III em países asiáticos e latinoamericanos (incluindo o Brasil) envolvendo mais de 30 mil participantes com idade entre dois e 14 anos (estudo "CYD14") e nove a 16 anos (estudo "CYD15") que receberam três doses ${ }^{43,44}$. As contraindicações estipuladas pelo fabricante são as mesmas encontradas para a maioria das vacinas atenuadas e incluem indivíduos com alergias a qualquer um dos componentes da vacina (não há adjuvantes na formulação), indivíduos imunossuprimidos, gestantes ou lactantes. Reações adversas locais e sistêmicas ocorridas após a vacinação foram semelhantes às registradas por outras vacinas vivas atenuadas ${ }^{45}$.

Tabela 2. Eventos adversos relacionados a outros componentes vacinais.

\begin{tabular}{|c|c|c|c|c|c|}
\hline Componente & Função na vacina & Vacina & Dose limite & Eventos adversos estudados & Referências \\
\hline Albumina (recombinante) & Estabilizante & Varicela/Tríplice viral & 50 ng/dose & Anafilaxia & 8 \\
\hline Antibióticos & $\begin{array}{l}\text { Evitar contaminação no } \\
\text { processo de fabricação }\end{array}$ & Febre amarela, influenza, pólio & NA & Reações alérgicas & 8 \\
\hline Formaldeído/ Glutaraldeído & $\begin{array}{l}\text { Inativação de toxinas ou } \\
\text { partículas virais }\end{array}$ & Influenza & $\begin{array}{l}200 \mathrm{ppm} \\
\text { (residual) }\end{array}$ & eczema, câncer & 8,29 \\
\hline Gelatina & Estabilizante & Febre amarela & $10 \mu \mathrm{L} / \mathrm{mL}$ & $\begin{array}{l}\text { Alergia, anafilaxia, } \\
\text { hipersensibilidade }\end{array}$ & $8,31,39$ \\
\hline Proteínas do ovo & Cultivo de vírus & Febre amarela, influenza & NA & Alergia, anafilaxia & 8,32 \\
\hline Sorbitol & Estabilizante & $\begin{array}{c}\text { Febre amarela, HPV, } \\
\text { pneumocócica conjugada, pólio }\end{array}$ & NA & Intolerância, alergia & 8 \\
\hline Timerosal & Conservante & $\begin{array}{l}\text { Tríplice bacteriana (DTP), tríplice } \\
\text { viral, Hepatite B, influenza }\end{array}$ & $\begin{array}{c}0,01 \% \text { ou } 25 \\
\mu \mathrm{g} \mathrm{Hg} / \text { dose }\end{array}$ & Alergia, TEA & $8,35-38,40$ \\
\hline
\end{tabular}

NA: não se aplica; TEA: Transtorno do Espectro Autista; HPV: vírus do papiloma humano 
No entanto, o grupo de vacinados com idade entre dois a cinco anos apresentou maior risco de hospitalização com formas graves da dengue em relação ao grupo placebo da mesma faixa etária, quando infectados pelo vírus selvagem em testes feitos na Ásia ${ }^{44}$. Esse efeito foi observado principalmente no terceiro ano após a primeira dose e não foi observado em outros grupos de idade (6-8, 9-11 e 12-16 anos). Além disso, uma estimativa global contra todos os sorotipos do vírus resultou em uma eficácia final de aproximadamente $60 \%$, mas uma eficácia de $33,7 \%$ para o grupo mais jovem $(2-5 \text { anos })^{45}$. Tais observações sugerem que a vacinação pode contribuir para uma infecção secundária exacerbada em crianças soronegativas muito jovens - fenômeno conhecido como ADE (Antibody Dependent Enhancement) ou aumento da replicação viral dependente de anticorpos. Nesse fenômeno, ocorre um aumento na replicação viral em indivíduos vacinados ou infectados com cepas virais diferentes daquelas previamente expostas, por meio da presença de anticorpos não neutralizantes. Portanto, a vacina foi indicada apenas para crianças maiores de nove anos.

\section{Riscos Associados à não Vacinação}

Dentre os riscos relacionados a vacinas, considera-se como o mais importante a não vacinação. Os efeitos deletérios associados ao uso de vacinas, quando presentes e comprovados cientificamente, ocorrem em frequência muito baixa e mostram-se inexpressivos quando comparados aos riscos relacionados a não vacinação. Estratégias de estímulo ao uso de vacinas são tradicionalmente adotadas em saúde pública, porém podem ser insuficientes para garantir aumento na cobertura vacinal. Nesse contexto, faz-se necessário manter uma compreensão clara sobre o valor das vacinas tanto na população, como entre os profissionais de saúde ${ }^{46}$. No Brasil, a vacinação é obrigatória e regulada por legislação federal (Decreto 78.231, de 12 de agosto de 1976) ${ }^{47}$.

No entanto, a decisão da não vacinação é individual e influenciada por fatores, como políticas de saúde pública, recomendação de profissionais de saúde, meios de comunicação e fatores intrínsecos ao indivíduo, tais como conhecimento e informação, experiências passadas, percepção da importância da vacinação e convicções morais e religiosas. Esses fatores estão inseridos em um contexto histórico, político e social que também deve ser considerado ${ }^{48}$. Entretanto, a decisão do indivíduo não acarreta consequências apenas para ele. A decisão de não se vacinar ou persuadir pessoas de seu convívio a não fazê-lo contribui para reduzir a imunidade populacional (ou imunidade de rebanho), podendo resultar em surtos localizados ou bolsões de infecção em grupos ou populações específicas. Esse tipo de situação tem assumido proporções preocupantes, principalmente a partir das décadas de 1970 e 1980, quando houve um aumento exponencial de casos de coqueluche em países desenvolvidos, uma doença facilmente controlada por meio de uma cobertura vacinal adequada ${ }^{49}$.

Esses surtos são cada vez mais frequentes e podem estar relacionados a vários fatores (Tabela 3). A maioria dos estudos corresponde a indivíduos que não se vacinaram por decisão individual ou que viajaram ou migraram de um ambiente com alta cobertura vacinal para outro com baixa cobertura vacinal, expondo populações não vacinadas ao patógeno. Devido a esse fenômeno, algumas doenças previamente controladas por programas eficazes de vacinação, como sarampo, ressurgiram em populações de diferentes partes do mundo, inclusive no Brasil.

Em relação à febre amarela, mais de 100 milhões de pessoas foram vacinadas na África Ocidental durante 2015. No entanto, um surto desta doença atingiu Angola e a República Democrática do Congo entre dezembro de 2015 e janeiro de 2016. Cerca de 70\% dos casos ocorreram em homens ${ }^{50,51}$. Estudos relacionaram esse surto com a alta densidade populacional aliada à baixa cobertura vacinal dos homens. No mesmo ano, foram relatados nove casos fatais de febre amarela, cinco no Brasil, todos em pessoas não vacinadas, mas em situações com recomendação da vacinação (turismo ou residentes em áreas rurais) ${ }^{52}$. Ainda, segundo o Centro de Operações de Emergências em Saúde Pública (COES), foram registrados 371 casos e 127 óbitos de 2016 a março de 2017. Especula-se a relação do surto com a baixa cobertura 
Tabela 3. Artigos científicos publicados entre 2010-2016, que correlacionam surtos de doenças infecciosas a indivíduos não vacinados e seus respectivos motivos/fontes dos surtos.

\begin{tabular}{|c|c|c|}
\hline Doença & País do surto (ano) $)^{\text {Motivo/fonte do surto }}$ & Referências \\
\hline Sarampo & 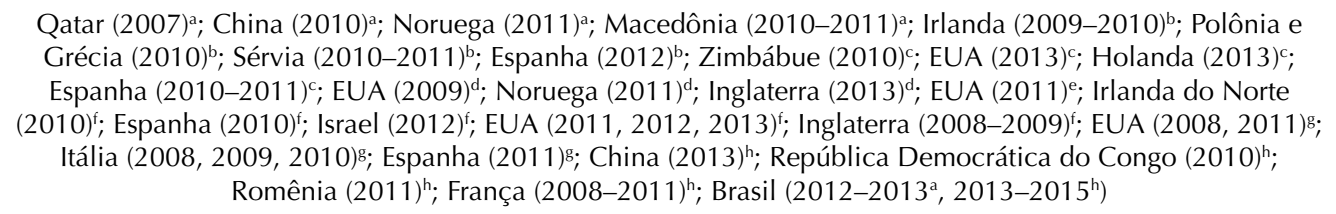 & 54-86 \\
\hline Caxumba & 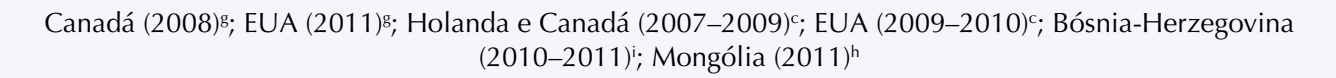 & $87-92$ \\
\hline Difteria & Noruega $(2008)^{\mathrm{g}}$ & 93 \\
\hline Rubéola & Romênia (2011-2012)g; Japão (2012-2013)s; China (2010-2011)a & 94,95 \\
\hline Coqueluche & Brasil $(2004,2007)^{\mathrm{h}} ;$ EUA $(2009-2010)^{\mathrm{c}} ;$ EUA $(2010,2012)^{\mathrm{g}}$; Itália (2009) ${ }^{\mathrm{h}}$; Papua Nova-Guiné (2011)i & 96-102 \\
\hline Febre amarela & Brasil (2008-2009) e República Democrática do Congo $(2015-2016)^{\mathrm{h}}$ & 50-52,103 \\
\hline Poliomielite & Somália $(2013)^{i}$ & 104 \\
\hline
\end{tabular}

a Migração/Imigração de indivíduos não vacinados.

${ }^{\mathrm{b}}$ Comunidades ciganas.

${ }^{c}$ Comunidades ortodoxas.

d Profissionais de saúde não vacinados.

e Refugiados.

${ }^{\mathrm{f}}$ Viajantes não vacinados.

$\mathrm{g}$ Indivíduos intencionalmente não vacinados.

h Falhas de vacinação/Acúmulo de não vacinados.

i Conflitos armados.

' Isolamento geográfico.

vacinal (por incluir regiões não endêmicas) e alguns fatores como desmatamento e acidentes ambientais em habitats de animais selvagens, incluindo hospedeiros primatas não humanos.

O sarampo foi considerado eliminado nas Américas desde 2002, mas apresenta incidência crescente no Brasil e no mundo, um reflexo da não vacinação voluntária. Entre 2013 e 2015, foram relatados mais de 1.000 casos só nos estados de Pernambuco e Ceará, afetando indivíduos entre 15 e 29 anos (34\%) e em bebês menores de um ano (27,5\%), devido à circulação de um tipo viral proveniente da Europa ${ }^{53,54}$. De acordo com a OMS, a estimativa é que a imunização tenha prevenido mais de 20 milhões de mortes entre 2000 e 2015 no mundo, tornando a vacina contra o sarampo uma das mais eficazes em saúde pública.

\section{CONSIDERAÇÕES FINAIS}

Os riscos associados ao uso de vacinas disponíveis não justificam a interrupção de qualquer formulação disponível no mercado. Por outro lado, o risco associado a "não vacinação" causa preocupações crescentes em diversos países. Campanhas publicitárias, disseminadas em mídias sociais ou mesmo revestidas de evidências supostamente "científicas" contribuem para o ressurgimento de doenças outrora erradicadas em grande parte do mundo. No Brasil, em particular, a falta de informações e a divulgação de informações não gabaritadas colaboram para o reaparecimento de doenças infecciosas, como o sarampo e a coqueluche. Destaca-se também o risco associado a não aceitação de vacinas, como os que envolvem as vacinas que previnem a infecção pelo HPV, cujos impactos esperados sobre a mortalidade deverão ocorrer somente se mantidas as condições adequadas de administração e cobertura vacinal. O papel dos profissionais de saúde na divulgação dos benefícios associados à vacinação é um dos mais importantes para que se possa assegurar saúde e qualidade de vida para a população. 


\section{REFERÊNCIAS}

1. Levi GC, Kallás EG. Varíola, sua prevenção vacinal e ameaça como agente de bioterrorismo. Rev Assoc Med Bras. 2002;48(4):357-62. https://doi.org/10.1590/S0104-42302002000400045

2. Miller ER, Moro PL, Cano M, Shimabukuro TT. Deaths following vaccination. What does the evidence show? Vaccine. 2015;33(29):3288-92. https://doi.org/10.1016/j.vaccine.2015.05.023

3. Murphy TV, Gargiullo PM, Massoudi MS, Nelson DB, Jumaan AO, Okoro CA, et al. Intussusception among infants given an oral rotavirus vaccine. $N$ Eng/ / Med. 2001;344(8):564-72. https://doi.org/10.1056/NE JM200102223440804

4. Ministério da Saúde (BR), Secretaria de Vigilância em Saúde, Departamento de Vigilância das Doenças Transmissíveis. Manual de vigilância epidemiológica de eventos adversos pósvacinação. 3.ed. Brasília (DF); 2014 [citado 20 nov 2017]. Disponível em: http://bvsms.saude. gov.br/bvs/publicacoes/manual_vigilancia_epidemiologica_eventos_adversos_pos_vacinacao.pdf

5. Stewart GT. Toxicity of pertussis vaccine: frequency and probability of reactions. J Epidemiol Community Health. 1979;33(2):150-6. https://doi.org/:10.1136/jech.33.2.150

6. Kimura M, Hikino N. Results with a new DTP vaccine in Japan. Dev Biol Stand. 1985;61:545-61.

7. Ministério da Saúde (BR). Calendário Nacional de Vacinação. Brasília (DF); 2014 [citado 20 nov 2017]. Disponível em: http://portalsaude.saude.gov.br/index.php/o-ministerio/principal/leiamais-o-ministerio/197-secretaria-svs/13600-calendario-nacional-de-vacinacao

8. Ministério da Saúde (BR), Secretaria de Vigilância em Saúde, Departamento de Vigilância Epidemiológica. Manual de vigilância epidemiológica de eventos adversos pós-vacinação. 2.ed. Brasília (DF); 2008 [citado 20 nov 2017]. Disponível em: http://bvsms.saude.gov.br/bvs/ publicacoes/manual_pos-vacinacao.pdf

9. Ministério da Saúde (BR), Secretaria de Vigilância em Saúde. Programa Nacional de Imunizações: aspectos históricos dos calendários de vacinação e avanços dos indicadores de coberturas vacinais, no período de 1980 a 2013. Bol Epidemiol. 2015 [citado 20 nov 2017];46(30):1-13. Disponível em: http://portalarquivos.saude.gov.br/images/pdf/2015/ outubro/14/besvs-pni-v46-n30.pdf13

10. Kata A. Anti-vaccine activists, Web 2.0, and the postmodern paradigm: an overview of tactics and tropes used online by the anti-vaccination movement. Vaccine. 2011;30(25):3778-89. https://doi.org/10.1016/j.vaccine.2011.11.112

11. Andrews N, Miller E, Waight P, Farrington P, Crowcroft N, Stowe J, et al. Does oral polio vaccine cause intussusception in infants? Evidence from a sequence of three selfcontrolled cases series studies in the United Kingdom. Eur J Epidemiol. 2001;17(8):701-6. https://doi.org/10.1023/A:1015691619745

12. Farrington $\mathrm{CP}$, Miller E, Taylor B. MMR and autism: further evidence against a causal association. Vaccine. 2001;19(27):3632-5. https://doi.org/10.1016/S0264-410X(01)00097-4

13. Miller E, Waight P, Farrington CP, Andrews N, Stowe J, Taylor B. Idiopathic thrombocytopenic purpura and MMR vaccine. Arch Dis Child. 2001;84(3):227-9. https://doi.org/10.1136/adc.84.3.227

14. O'Hagan DT, De Gregorio E. The path to a successful vaccine adjuvant: 'the long and winding road'. Drug Discov Today. 2009;14(11-12):541-51. https://doi.org/10.1016/j.drudis.2009.02.009

15. Shoenfeld Y, Agmon-Levin N. 'ASIA' - autoimmune/inflammatory syndrome induced by adjuvants. J Autoimmun. 2011;36(1):4-8. https://doi.org/10.1016/j.jaut.2010.07.003

16. Batista-Duharte A, Lastre M, Pérez O. Adyuvantes inmunológicos. Determinantes en el balance eficacia-toxicidad de las vacunas contemporáneas. Enferm Infec Microbiol Clin. 2014;32(2):106-14. https://doi.org/10.1016/j.eimc.2012.11.012

17. Becaria A, Campbell A, Bondy SC. Aluminum as a toxicant. Toxicol Ind Health. 2002;18(7):309-20. https://doi.org/10.1191/0748233702th157oa

18. Israeli E, Agmon-Levin N, Blank M, Shoenfeld Y. Adjuvants and autoimmunity. Lupus. 2009;18(13):1217-25. https://doi.org/10.1177/0961203309345724

19. Laan JW, Gould S, Tanir JY; ILSI HESI Vaccines and Adjuvants Safety Project Committee. Safety of vaccine adjuvants: focus on autoimmunity. Vaccine. 2015;33(13):1507-14. https://doi.org/10.1016/j.vaccine.2015.01.073

20. Guimarães LE, Baker B, Perricone C, Shoenfeld Y. Vaccines, adjuvants and autoimmunity. Pharmacol Res. 2015;100:190-209. https://doi.org/10.1016/j.phrs.2015.08.003 
21. Siegrist CA. Godeau P, Canlorbe P, Nezelof C, Paolaggi J, Polonovski J, et al. Les adjuvants vaccinaux et la myofasciite à macrophages. Bull Acad Natl Med. 2003 [citado 20 nov 2017];187(8):1511-21. Disponível em: http://www.academie-medecine.fr/publication100035129/

22. Brinth LS, Pors K, Theibel AC, Mehlsen J. Orthostatic intolerance and postural tachycardia syndrome as suspected adverse effects of vaccination against human papilloma virus. Vaccine. 2015;33(22):2602-5. https://doi.org/10.1016/j.vaccine.2015.03.098

23. Martínez-Lavín M, Martínez-Martínez LA, Reyes-Loyola P. HPV vaccination syndrome: a questionnaire-based study. Clin Rheumatol. 2015;34(11):1981-3. https://doi.org/10.1007/s10067-015-3070-3

24. Tomljenovic L, Colafrancesco S, Perricone C, Shoenfeld Y. Postural orthostatic tachycardia with chronic fatigue after HPV vaccination as part of the "Autoimmune/Auto-inflammatory Syndrome Induced by Adjuvants": case report and literature review. I Investig Med High Impact Case Rep. 2014;2(1):2324709614527812. https://doi.org/10.1177/2324709614527812

25. Souayah N, Michas-Martin PA, Nasar A, Krivitskaya N, Yacoub HA., Khan H, et al. GuillainBarré syndrome after Gardasil vaccination: data from Vaccine Adverse Event Reporting System 2006-2009. Vaccine. 2011;29(5):886-9. https://doi.org/10.1016/j.vaccine.2010.09.020

26. Lippi G, Targher G, Franchini M. Vaccination, squalene and anti-squalene antibodies: facts or fiction? Eur J Intern Med. 2010;21(2):70-3. https://doi.org/10.1016/j.ejim.2009.12.001

27. Nohynek H, Jokinen J, Partinen M, Vaarala O, Kirjavainen T, Sundman J, et al. AS03 adjuvanted AH1N1 vaccine associated with an abrupt increase in the incidence of childhood narcolepsy in Finland. PLoS One. 2012;7(3):e33536. https://doi.org/10.1371/journal.pone.0033536

28. De Serres G, Rouleau I, Skowronski DM, Ouakki M, Lacroix K, Bédard F, et al. Paresthesia and sensory disturbances associated with 2009 pandemic vaccine receipt: clinical features and risk factors. Vaccine. 2015;33(36):4464-71. https://doi.org/10.1016/j.vaccine.2015.07.028

29. Mitkus RJ, Hess MA, Schwartz SL. Pharmacokinetic modeling as an approach to assessing the safety of residual formaldehyde in infant vaccines. Vaccine. 2013;31(25):2738-43. https://doi.org/10.1016/j.vaccine.2013.03.071

30. Ring J. Exacerbation of eczema by formalin-containing hepatitis B vaccine in formaldehydeallergic patient. Lancet. 1986;328(8505):522-3. https://doi.org/10.1016/S0140-6736(86)90397-1

31. Saito A, Kumagai T, Kojima H, Terai I, Yamanaka T, Wataya Y, et al. A sero-epidemiological survey of gelatin sensitization in young Japanese children during the 1979-1996 period. Scand J Immunol. 2005;61(4):376-9. https://doi.org/10.1111/j.1365-3083.2005.01590.x

32. Nagao M, Fujisawa T, Ihara T, Kino Y. Highly increased levels of IgE antibodies to vaccine components in children with influenza vaccine-associated anaphylaxis. J Allergy Clin Immunol. 2016;137(3):861-7. https://doi.org/10.1016/j.jaci.2015.08.001

33. Wakefield AJ, Murch SH, Anthony A, Linnell J, Casson DM, Malik M, et al. Ileal-lymphoidnodular hyperplasia, non-specific colitis, and pervasive developmental disorder in children: early report. Lancet. 1998;351(9103):637-41. Retraction in: The editors of Lancet. Lancet. 2010:375(9713):445. https://doi.org/10.1016/S0140-6736(97)11096-0

34. Caplan AL. Retraction: Ileal-lymphoid-nodular hyperplasia, non-specific colitis, and pervasive disorder in children. Lancet. 2010;375(9713):445. https://doi.org/10.1016/S0140-6736(10)60175-4

35. Burbacher TM, Shen DD, Liberato N, Grant KS, Cernichiari E, Clarkson T. Comparison of blood and brain mercury levels in infant monkeys exposed to methylmercury or vaccines containing thimerosal. Environ Health Perspect. 2005;113(8):1015-21. https://doi.org/10.1289/ehp.7712

36. Madsen KM, Hviid A, Vestergaard M, Schendel D, Wohlfahrt J, Thorsen P, et al. A Populationbased study of measles, mumps, and rubella vaccination and autism. N Engl J Med. 2002;347(19):1477-82. https://doi.org/10.1056/NEJMoa021134

37. Honda H, Shimizu Y, Rutter M. No effect of MMR withdrawal on the incidence of autism: a total population study. J Child Psychol Psychiatry. 2005;46(6):572-9. https://doi.org/10.1111/j.1469-7610.2005.01425.x

38. Bernard S. Association between thimerosal-containing vaccine and autism. JAMA. 2004;291(2):180. https//doi.org/10.1001/jama.291.2.180-b

39. Pool V, Braun MM, Kelso JM, Mootrey G, Chen RT, Yunginger JW, et al. Prevalence of anti-gelatin IgE antibodies in people with anaphylaxis after measles-mumps rubella vaccine in the United States. Pediatrics. 2002;110(6):e71. https://doi.org/10.1542/peds.110.6.e71 
40. Nokleby H. Vaccination and anaphylaxis. Curr Allergy Asthma Rep. 2006;6(1):9-13. https://doi.org/10.1007/s11882-006-0003-x

41. World Health Organization. Human papillomavirus vaccines: WHO position paper, October 2014. Wkly Epidemiol Rec. 2014 [citado 20 nov 2017];89(43):465-92. http://www.who.int/ wer/2014/wer8943.pdf

42. Ferguson NM, Rodríguez-Barraquer I, Dorigatti I, Mier-y-Teran-Romero L, Laydon DJ, Cummings DAT. Benefits and risks of the Sanofi-Pasteur dengue vaccine: modeling optimal deployment. Science. 2016;353(6303):1033-6. Disponível em: https://doi.org/10.1126/science.aaf9590

43. Villar L, Dayan GH, Arredondo-García JL, Rivera DM, Cunha R, Deseda C, et al. Efficacy of a tetravalent dengue vaccine in children in Latin America. N Engl J Med. 2015;372(2):113-23. https://doi.org/10.1056/NEJMoa1411037

44. Capeding MR, Tran NH, Hadinegoro SRS, Ismail HIHJM, Chotpitayasunondh T, Chua $M N$, et al. Clinical efficacy and safety of a novel tetravalent dengue vaccine in healthy children in Asia: a phase 3, randomised, observer-masked, placebo-controlled trial. Lancet. 2014;384(9951):1358-65. https://doi.org/10.1016/S0140-6736(14)61060-6

45. World Health Organization. Dengue vaccine: WHO position paper - July 2016. Wkly Epidemiol Rec. 2016 [citado 20 nov 2017];91(30):349-64. Disponível em: http://www.who.int/wer/2016/ wer9130.pdf?ua $=1$

46. Colgrove J. Vaccine refusal revisited: the limits of public health persuasion and coercion. N Eng/ J Med. 2016;375(14):1316-7. https://doi.org/10.1056/NEJMp1608967

47. Levi GC. Recusa de vacinas: causas e consequências. São Paulo: Segmento Farma; 2013.

48. Dubé E, Laberge C, Guay M, Bramadat P, Roy R, Bettinger J. Vaccine hesitancy: an overview. Hum Vaccines Immunother. 2013;9(8):1763-73. https://doi.org/10.4161/hv.24657

49. Gangarosa EJ, Galazka AM, Wolfe CR, Phillips LM, Gangarosa RE, Miller E, et al. Impact of anti-vaccine movements on pertussis control: the untold story. Lancet. 1998;351(9099):356-61. https://doi.org/10.1016/S0140-6736(97)04334-1

50. Marlow MA, Pambasange MA, Francisco C, Receado OD, Soares MJ, Silva S, et al. Notes from the field: knowledge, attitudes, and practices regarding yellow fever vaccination among men during an outbreak - Luanda, Angola, 2016. MMWR Morb Mortal Wkly Rep. 2017;66(4):117-8. https://doi.org/ 10.15585/mmwr.mm6604a6

51. Kraemer MUG, Faria NR, Reiner RC Jr, Golding N, Nikolay B, Stasse S, et al. Spread of yellow fever virus outbreak in Angola and the Democratic Republic of the Congo 2015-16: a modelling study. Lancet Infect Dis. 2016;17(3):330-8. https://doi.org/10.1016/S1473-3099(16)30 S513-8

52. Nishino K, Luce R, Mendez Rico JA, Garnier, Millot V, Garcia E, et al. Yellow fever in Africa and South America, 2015. Wkly Epidemiol Rec. 2016 [citado 20 nov 2017];91(32):381-8. Disponível em: http://www.who.int/wer/2016/wer9132.pdf?ua=1

53. Leite RD, Barreto JLTMS, Sousa AQ. Measles reemergence in Ceará, Northeast Brazil, 15 years after elimination. Emerg Infect Dis. 2015;21(9):1681-3. https://doi.org/10.3201/eid2109.150391

54. Leite RD, Berezin EN. Measles in Latin America: current situation. J Pediatric Infect Dis Soc. 2015;4(3):179-81. https://doi.org/10.1093/jpids/piv047

55. Gao J, He H, Shen J, Huang Z, Ma H, Luo S, et al. [An outbreak of measles among unvaccinated migrant population in Zhejiang province, from June to August, 2010]. Zhonghua Liu Xing Bing Xue Za Zhi. 2010;31(10):1163-5. Chinese. https://doi.org/10.3760/cma.j.issn.0254-6450.2010.10.020

56. Vainio K, Ronning K, Steen TW, Arnesen TM, Ånestad G, Dudman S. Ongoing outbreak of measles in Oslo, Norway, January-February 2011. Euro Surveill. 2011;16(8):4-6. https://doi.org/10.2807/ese.16.08.19804-en

57. Al-Kuwari MG, Nazzal ZA, Al Khenji AA. Epidemiology of measles outbreaks in Qatar in 2007. East Mediterr Health J. 2011;17(3):186-90.

58. Kondova IT, Milenkovic Z, Marinkovic SP, Bosevska G, Kuzmanovska G, Kondov $\mathrm{G}$, et al. Measles outbreak in Macedonia: epidemiological, clinical and laboratory findings and identification of susceptible cohorts. PLoS One. 2013;8(9):e74754. https://doi.org/10.1371/journal.pone.0074754

59. Pervanidou D, Horefti E, Patrinos S, Lytras T, Triantafillou E, Mentis A., et al. Spotlight on measles 2010: ongoing measles outbreak in Greece, January-July 2010. Euro Surveill. 2010;15(30). https://doi.org/10.2807/ese.15.30.19629-en 
60. Rogalska J, Santibanez S, Mankertz A, Makowka A., Szenborn L, Stefanoff P. Spotlight on measles 2010: an epidemiological overview of measles outbreaks in Poland in relation to the measles elimination goal. Euro Surveill. 2010;15(17):20-5. https://doi.org/10.2807/ese.15.30.19629-en

61. Gee S, Cotter S, O'Flanagan D; National Incident Management Team. Spotlight on measles 2010: measles outbreak in Ireland 2009-2010. Euro Surveill. 2010;15(9):2-5. https://doi.org/10.2807/ese.15.09.19500-en

62. Martínez-Ramírez M, González-Praetorius A, Ory-Manchón F, Martínez-Benito Y, García-Rivera MV, Hübschen J, et al. Reemergencia de sarampión en la provincia de Guadalajara. ¿Es el momento de establecer nuevas estrategias para su eliminación? Enferm Infecc Microbiol Clin. 2014;32(8):486-90. https://doi.org/10.1016/j.eimc.2013.08.005

63. Nedeljković J, Rakić Adrović S, Tasić G, Kovaćević-Jovanović V, Lončarević G, Hübschen JM, et al. Resurgence of measles in Serbia 2010-2011 highlights the need for supplementary immunization activities. Epidemiol Infect. 2016;144(5):1121-8. https://doi.org/10.1017/S0950268815002277

64. Pomerai KW, Mudyiradima RF, Gombe NT. Measles outbreak investigation in Zaka, Masvingo Province, Zimbabwe, 2010. BMC Res Notes. 2012;5(1):687. https://doi.org/10.1186/1756-0500-5-687

65. Navarro E, Mochón MM, Galicia MD, Marín I, Laguna J. Study of a measles outbreak in Granada with preventive measures applied by the courts, Spain, 2010 to 2011. Euro Surveill. 2013;18(43). https://doi.org/10.2807/1560-7917.ES2013.18.43.20612

66. Centers for Disease Control and Prevention. Prepregnancy contraceptive use among teens with unintended pregnancies resulting in live births - Pregnancy Risk Assessment Monitoring System (PRAMS), 2004-2008. MMWR Morb Mortal Wkly Rep. 2012 [citado 20 nov 2017];61(2):25-9. Disponível em: https://www.cdc.gov/mmwr/preview/mmwrhtml/mm6102a1.htm

67. Centers for Disease Control and Prevention. Notes from the field: measles outbreak among members of a religious community - Brooklyn, New York, March-June 2013. MMWR Morb Mortal Wkly Rep. 2013 [citado 20 nov 2017];62(36):752-3. Disponível em: https://www.cdc. gov/mmwr/preview/mmwrhtml/mm6236a5.htm

68. Centers for Disease Control and Preventon. Hospital-associated aeasles outbreak - Pennsylvania, March-April 2009. MMWR Morb Mortal Wkly Rep. 2012 [citado 20 nov 2017];61(2):30-2. Disponível em: https://www.cdc.gov/mmwr/preview/mmwrhtml/mm6102a2.htm

69. Vainio K, Steen TW, Arnesen TM, Rønning K, Ånestad G, Dudman S. Measles virus genotyping an important tool in measles outbreak investigation in Norway, 2011. Euro Surveill. 2012;17(50). https://doi.org/10.2807/ese.17.50.20340-en

70. Baxi R, Mytton OT, Abid M, Maduma-Butshe A, lyer S, Ephraim A, et al. Outbreak report: nosocomial transmission of measles through an unvaccinated healthcare worker-implications for public health. J Public Health (Oxf). 2014;36(3):375-81. https://doi.org/10.1093/pubmed/fdt096

71. Smithson R, Irvine N, Hutton C, Doherty L, Watt A. Spotlight on measles 2010: ongoing measles outbreak in Northern Ireland following an imported case, September-October 2010. Euro Surveill. 2010;15(43):1-4. https://doi.org/10.2807/ese.15.43.19698-en

72. López Hernández B, Laguna Sorinas J, Marín Rodríguez I, Gallardo García V, Pérez Morilla E, Mayoral Cortés JM. Spotlight on measles 2010: an ongoing outbreak of measles in an unvaccinated population in Granada, Spain, October to November 2010. Euro Surveill. 2010;15(50):3-6. https://doi.org/10.2807/ese.15.50.19746-en

73. Kopel E, Amitai Z, Savion M, Aboudy Y, Mendelson E, Sheffer R. Ongoing African measles virus genotype outbreak in Tel Aviv district since April, Israel, 2012. Euro Surveill. 2012;17(37). https:// doi.org/10.2807/ese.17.37.20272-en

74. Ghebrehewet S, Hayhurst G, Keenan A, Moore H. Outbreak of measles in Central and Eastern Cheshire, UK, October 2008-February 2009. Epidemiol Infect. 2012:14(9):1849-56. https://doi. org/10.1017/S0950268812002300

75. Centers for Disease Control and Prevention. Two measles outbreaks after importation - Utah, March-June 2011. MMWR Morb Mortal Wkly Rep. 2013 [citado 20 nov 2017];62(12):222-5. Disponível em: https://www.cdc.gov/mmwr/preview/mmwrhtml/mm6212a2.htm

76. Gahr P, DeVries AS, Wallace G, Miller C, Kenyon C, Sweet K, et al. An outbreak of measles in an undervaccinated community. Pediatrics. 2014;134(1):e220-8. https://doi.org/10.1542/peds.2013-4260

77. Slade TA, Klekamp B, Rico E, Mejia-Echeverry A. Measles outbreak in an unvaccinated family and a possibly associated international traveler - Orange County, Florida, December 2012-January 2013. MMWR Morb Mortal Wkly Rep. 2014 [citado 20 nov 2017];63(36):781-4. Disponível em: https://www.cdc.gov/mmwr/preview/mmwrhtml/mm6336a1.htm 
78. Sugerman DE, Barskey AE, Delea MG, Ortega-Sanchez IR, Bi D, Ralston KJ, et al. Measles outbreak in a highly vaccinated population, San Diego, 2008: role of the intentionally undervaccinated. Pediatrics. 2010;125(4):747-55. https://doi.org/10.1542/peds.2009-1653

79. Bassetti M, Schenone E, Calzi A, Camera M, Valle L, Ansaldi F, et al. Measles outbreak in adults in Italy. Infez Med. 2011 [citado 20 nov 2017];19:16-9. Disponível em: http://www.infezmed.it/ media/journal/Vol_19_1_2011_2.pdf

80. Filia A, Tavilla A, Bella A, Magurano F, Ansaldi F, Chironna M, et al. Measles in Italy, July 2009 to September 2010. Euro Surveill. 2011;16(29). https://doi.org/10.2807/ese.16.29.19925-en

81. Centers for Disease Control and Prevention. Notes from the field: measles outbreak - Indiana, June-July 2011. MMWR Morb Mortal Wkly Rep. 2011 [citado 20 nov 2017];60(34):1169. Disponível em: https://www.cdc.gov/mmwr/preview/mmwrhtml/mm6034a5.htm

82. Stanescu A, Janta D, Lupulescu E, Necula G, Lazar M, Molnar G, et al. Ongoing measles outbreak in Romania, 2011. Euro Surveill. 2011;16(31). https://doi.org/10.2807/ese.16.31.19932-en

83. Antona D, Lévy-Bruhl D, Baudon C, Freymuth F, Lamy M, Maine C, et al. Measles elimination efforts and 2008-2011 outbreak, France. Emerg Infect Dis. 2013;19(3):357-64. https://doi.org/10.3201/eid1903.121360

84. Zheng X, Zhang N, Zhang X, Hao L, Su Q, Wang H, et al. Investigation of a measles outbreak in China to identify gaps in vaccination coverage, routes of transmission, and interventions. PLoS One. 2015;10(7):e0133983. https://doi.org/10.1371/journal.pone.0133983

85. Scobie HM, Ilunga BK, Mulumba A, Shidi C, Coulibaly T, Obama R, et al. Antecedent causes of a measles resurgence in the Democratic Republic of the Congo. Pan Afr Med J. 2015;21:30. https://doi.org/i:10.11604/pamj.2015.21.30.6335

86. Rocha HAL, Correia LL, Campos JS, Silva AC, Andrade FO, Silveira DI, et al. Factors associated with non-vaccination against measles in northeastern Brazil: clues about causes of the 2015 outbreak. Vaccine. 2015;33(38):4969-74. https://doi.org/10.1016/j.vaccine.2015.07.027

87. Tan KE, Anderson M, Krajden M, Petric M, Mak A, Naus M. Mumps virus detection during an outbreak in a highly unvaccinated population in British Columbia. Can J Public Health. 2011;102(1):47-50. https://doi.org/10.17269/cjph.102.2340

88. Centers for Disease Control and Prevention. Mumps outbreak on a University Campus California, 2011. MMWR Morb Mortal Wkly Rep. 2012 [citado 20 nov 2017];61(48):986-9. Disponível em: https://www.cdc.gov/mmwr/preview/mmwrhtml/mm6148a2.htm

89. Wielders CC, Binnendijk RS, Snijders BE, Tipples G a., Cremer J, Fanoy E, et al. Mumps epidemic in orthodox religious low-vaccination communities in the Netherlands and Canada, 2007 to 2009. Euro Surveill. 2011;16(41). https://doi.org/10.2807/ese.16.41.19989-en

90. Barskey AE, Schulte C, Rosen JB, Handschur EF, Rausch-Phung E, Doll MK, et al. Mumps outbreak in Orthodox Jewish communities in the United States. N Engl J Med. 2012;367(18):1704-13. https://doi.org/10.1056/NEJMoa1202865

91. Hukic M, Ravlija J, Ljubovic AD, Moro A., Arapcic S, Muller CP, et al. Ongoing large mumps outbreak in the Federation of Bosnia and Herzegovina, Bosnia and Herzegovina, December 2010 to July 2011. Euro Surveill. 2011;16(35):1-4. https://doi.org/10.2807/ese.16.35.19959-en

92. Munkhjargal I, Selenge J, Ambalselmaa A, Tuul R, Delgermaa P, Amarzaya S, et al. Investigation of a mumps outbreak in Mongolia, January to April 2011. West Pacific Surveill Response J. 2012;3(4):53-8. https://doi.org/10.5365/wpsar.2012.3.3.007

93. Rasmussen I, Wallace S, Mengshoel AT, Hoiby EA, Brandtzaeg P. Diphtheria outbreak in Norway: lessons learned. Scand J Infect Dis. 2011;43(11-12):986-9. https://doi.org/10.3109/00365548.2011.600326

94. Janta D, Stanescu A, Lupulescu E, Molnar G, Pistol A. Ongoing rubella outbreak among adolescents in Salaj, Romania, September 2011-January 2012. Euro Surveill. 2012;17(7):1-4. https://doi.org/10.2807/ese.17.07.20089-en

95. Sugishita Y, Takahashi T, Hori N, Abo M. Ongoing rubella outbreak among adults in Tokyo, Japan, June 2012 to April 2013. West Pac Surveill Response J. 2013;4(3):37-41. https://doi.org/10.5365/wpsar.2013.4.2.011

96. Cantey JB, Sánchez PJ, Tran J, Chung W, Siegel JD. Pertussis: a persistent cause of morbidity and mortality in young infants. J Pediatr. 2014;164(6):1489-93. https://doi.org/10.1016/j.jpeds.2014.01.023

97. Atwell JE, Van Otterloo J, Zipprich J, Winter K, Harriman K, Salmon DA, et al. Nonmedical vaccine exemptions and pertussis in California, 2010. Pediatrics. 2013;132(4):624-30. https://doi.org/10.1542/peds.2013-0878 
98. Tafuri S, Gallone MS, Martinelli D, Prato R, Chironna M, Germinario C. Report of a pertussis outbreak in a low coverage booster vaccination group of otherwise healthy children in Italy. BMC Infect Dis. 2013;13:541. https://doi.org/10.1186/1471-2334-13-541

99. Datta SS, Toikilik S, Ropa B, Chidlow G, Lagani W. Pertussis outbreak in Papua New Guinea: the challenges of response in a remote geo-topographical setting. West Pacific Surveill Response J. 2012;3(4):3-6. https://doi.org/10.5365/WPSAR.2012.3.3.008

100. Medina-Marino A, Reynolds D, Finley C, Hays S, Jones J, Soyemi K. Communication and mass vaccination strategies after pertussis outbreak in rural Amish communities-Illinois, 2009-2010. J Rural Health. 2013;29(4):413-9. https://doi.org/10.1111/jrh.12019

101. Druzian AF, Brustoloni YM, Oliveira SMVL, Matos VTG, Negri ACG, Pinto CS, et al. Pertussis in the central-west region of Brazil: one decade study. Braz J Infect Dis. 2014;18(2):177-80. https:// doi.org/10.1016/j.bjid.2013.08.006

102. Baptista PN, Magalhães VS, Rodrigues LC. The role of adults in household outbreaks of pertussis. Int J Infect Dis. 2010;14(2):e111-4. https://doi.org/10.1016/j.ijid.2009.03.026

103. Romano APM, Costa ZGA, Ramos DG, Andrade MA, Jayme VS, Almeida MAB, et al. Yellow Fever outbreaks in unvaccinated populations, Brazil, 2008-2009. PLoS Neg/ Trop Dis. 2014;8(3):e2740. https://doi.org/10.1371/journal.pntd.0002740

104. Kamadjeu R, Mahamud A, Webeck J, Baranyikwa MT, Chatterjee A, Bile YN, et al. Polio outbreak investigation and response in Somalia, 2013. J Infect Dis. 2014;210 Suppl 1:S181-6. https://doi.org/10.1093/infdis/jiu453

Financiamento: Fundação de Amparo à Pesquisa do Estado de São Paulo (Fapesp - Processo 2013/15360-2).

Contribuição dos Autores: Todos os autores participaram da concepção e planejamento do estudo, coleta, análise e interpretação dos dados e da aprovação da versão final. LRMMA, MAFP e LCSF realizaram a elaboração e revisão do manuscrito e assumem a responsabilidade pública pelo seu conteúdo. LRMMA e MAFP contribuíram igualmente para este trabalho.

Conflito de Interesses: Os autores declaram não haver conflito de interesses. 